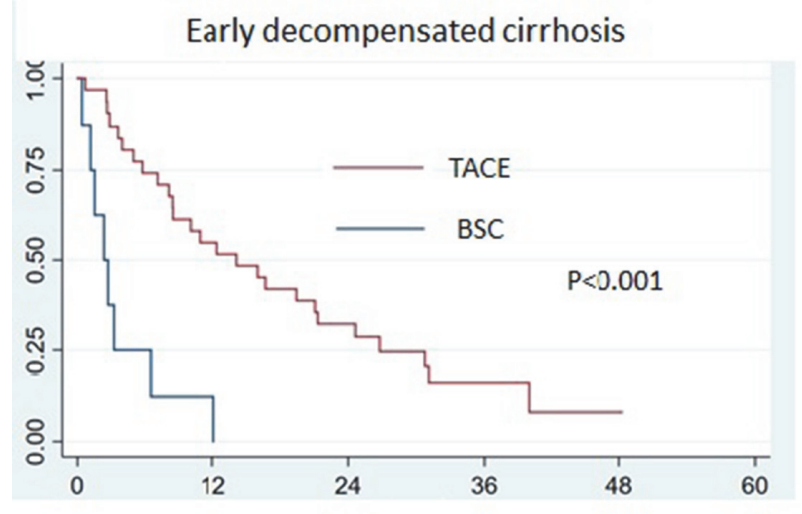

Abstract IDDF2021-ABS-0067 Figure 3

received BSC (21.4 VS 8.2 months, Log-rank $\mathrm{P}<0.001)$. Subgroup analyses showed patients undergoing TACE had increased median OS comparing to those who received BSC in both compensated cirrhosis (26.0 VS 9.0 months, Log-rank $\mathrm{P}<0.001)$ and early decompensated cirrhosis (14.5 VS 6.9 months, Log-rank $\mathrm{P}<0.001)$. In the Cox proportional-hazards model, undergoing TACE is an independent factor associated with prolonged OS in overall patients (Hazard ratio; HR 0.29, 95\% CI 0.17-0.49, $\mathrm{P}<0.001$ ), compensated cirrhosis (HR 0.31, 95\% CI 0.16-0.62, $\mathrm{P}<$ 0.001 ), and early decompensated cirrhosis (HR 0.16, 95\% CI 0.061-0.44, $\mathrm{P}<0.001)$.

(IDDF2021-ABS-0067 Figure 1, IDDF2021-ABS-0067 Figure 2, IDDF2021-ABS-0067 Figure 3)

Conclusions This study demonstrated the beneficial effect of TACE on OS in patients with intermediate-stage HCC who had compensated and early decompensated cirrhosis.

\section{IDDF2021-ABS-0070 PREDICTIVE PERFORMANCE OF THE HAP SCORE AT PATIENTS WITH HEPATOCELLULAR CARCINOMA FOLLOWING TRANS-ARTERIAL CHEMOEMBOLISATION (TACE)}

Anh Tran Ngoc*, Hieu Nguyen Trung, Lieu Dau Quang. Hanoi Medical University Hospital, Vietnam

\subsection{6/gutjnl-2021-IDDF.77}

Background Transarterial chemoembolization (TACE) is the therapy which have chosen in $50-60 \%$ of patients with Hepatocellular carcinoma (HCC). Recently, Hepatoma Arterial Embolization (HAP) score stratifies patients who will benefit from the first TACE. We conducted a survey to investigate the value of HAP score in the prognosis of HCC treated with TACE in Vietnam.

Methods Prospective cohort study in patients with HCC and first TACE at the Department of Internal Medicine, Hanoi Medical University Hospital, Vietnam, from January 2017 to June 2021. The HAP score (serum albumin $0.9 \mathrm{mg} / \mathrm{dl}$, alphafoetoprotein $>400 \mathrm{ng} / \mathrm{ml}$, and tumor size $>7 \mathrm{~cm}$ ), and ALBI score was applied before first TACE, mortality and survival were observed with a follow-up of 54 months.
Results We included 106 patients with age of $62.8 \pm 10.1$ years. In this population, HCC risk factor was mainly hepatitis B with 75.5\% (80/106). The HAP score classified 19, 41, 31 and 15 patients as HAP A, B, C and D, respectively,

Overall Comparisons

\begin{tabular}{|l|r|r|c|}
\hline & Chi-Square & df & $p$ \\
\hline Log Rank (Mantel-Cox) & 17.863 & 3 & $<0.001$ \\
\hline
\end{tabular}

Test of equality of survival distributions for the different levels of HAP.

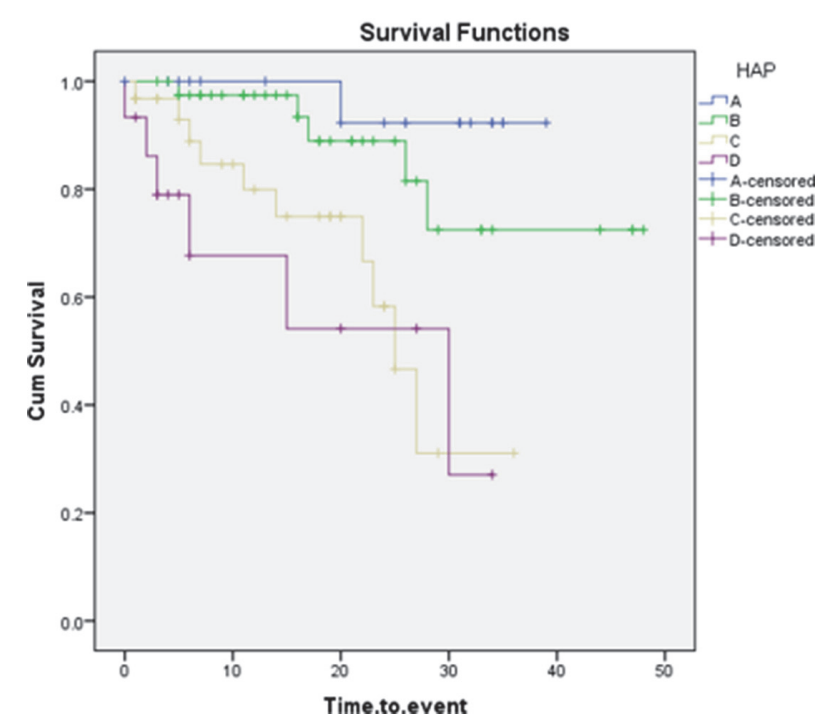

Abstract IDDF2021-ABS-0070 Figure 2 Kaplan-Meier curve for HAP score

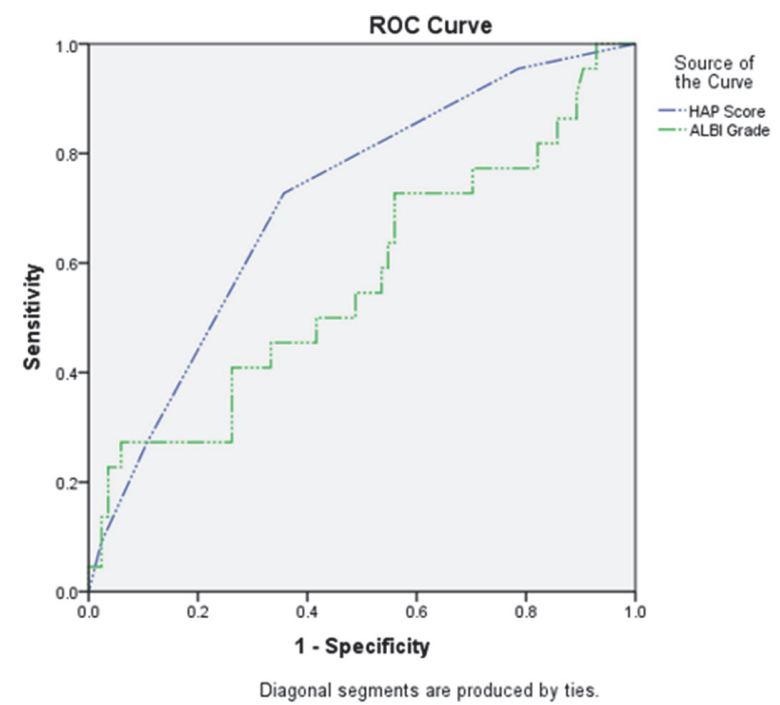

Area Under the Curve

\begin{tabular}{|l|c|}
\hline Test Result Variable(s) & Area \\
\hline HAP Score & .711 \\
ALBI.Diem & .568 \\
\hline
\end{tabular}

Abstract IDDF2021-ABS-0070 Figure 3 ROC curves for HAP score and ALBI grade 


\begin{tabular}{llll} 
Abstarct IDDF2021-ABS-0070 & Table 1 & Baseline characteristics \\
\hline & & $\mathbf{n}$ & Percentage \\
\hline Age (years) & Male & 97 & $62.8 \pm 10.1$ \\
Sex & Female & 9 & $91.5 \%$ \\
& HBV & 80 & $8.5 \%$ \\
Aetiology & HCV & 8 & $75.5 \%$ \\
& HBV+HCV & 1 & $7.5 \%$ \\
& Others & 17 & $0.9 \%$ \\
Serum & Albumin $\geq$ & 69 & $16.0 \%$ \\
albumin (g/1) & 36 & 37 & $65.1 \%$ \\
& Albumin $<36$ & & $34.9 \%$ \\
Total bilirubin & Bilirubin $\leq 17$ & 64 & \\
(mmol/l) & Bilirubin $>17$ & 42 & $60.4 \%$ \\
AFP (ng/ml) & AFP $\leq 400$ & 71 & $39.6 \%$ \\
& AFP $>400$ & 35 & $67 \%$ \\
Tumor size & $\leq 7$ & 68 & $33 \%$ \\
(cm) & $>7$ & 38 & $64.2 \%$ \\
HAP & HAP A & 19 & $35.8 \%$ \\
& HAP B & 41 & $17.9 \%$ \\
& HAP C & 31 & $38.7 \%$ \\
& HAP D & 15 & $29.2 \%$ \\
& & & $14.2 \%$ \\
\hline & & &
\end{tabular}

\begin{tabular}{|c|c|c|c|c|c|c|c|c|c|}
\hline \multicolumn{8}{|c|}{$\begin{array}{l}\text { Abstract IDDF2021-ABS-0070 Table } 2 \\
\text { surviving at the time }\end{array}$} & \multicolumn{2}{|c|}{ Cumulative proportion } \\
\hline \multirow[t]{2}{*}{ HAP } & \multicolumn{2}{|c|}{6 months } & \multicolumn{2}{|c|}{12 months } & \multicolumn{2}{|c|}{24 months } & \multicolumn{2}{|c|}{36 months } & \multirow{2}{*}{$\begin{array}{l}\text { Means for survival } \\
\text { time } \\
\text { (months) }\end{array}$} \\
\hline & $n$ & $(\%)$ & n & $(\%)$ & n & $(\%)$ & $\mathrm{n}$ & $(\%)$ & \\
\hline A & 0 & 100 & 0 & 100 & 1 & 92.3 & 1 & 92.3 & $37.5 \pm 1.2$ \\
\hline B & 1 & 97.4 & 1 & 97.4 & 3 & 88.9 & 5 & 72.5 & $40.8 \pm 2.9$ \\
\hline C & 3 & 88.9 & 5 & 79.9 & 8 & 58.3 & 10 & 31.1 & $24.0 \pm 2.5$ \\
\hline D & 4 & 66.7 & 4 & 66.7 & 5 & 54.2 & 6 & 27.1 & $20.4 \pm 4.1$ \\
\hline
\end{tabular}

(IDDF2021-ABS-0070 Table 1). The median follow-up time was $37.5 \pm 1.2$ months for HAP A, 40.8 \pm 2.9 months for HAP B, 24.0 \pm 2.5 months for HAP C and 20.4 \pm 4.1 months for HAP D. There were no deaths at the first year in HAP A. The cumulative proportion surviving at first years for HAP B, C, D was 97.4; 77.9 and 66.7, respectively. At 36 months, this percentage for HAP A, B, C and D was 92.3; 72.5; 31.1 and 27.1, respectively, (IDDF2021-ABS-0070 Table 2). Survival of all subgroups differed significantly from each other (each $\mathrm{p}$ $<$ 0.05) (IDDF2021-ABS-0070 Figure 2. Kaplan-Meier curve for HAP score). Area under the curve for receiver operating characteristic of HAP score was 0.71 and higher than this of ALBI grade (0.57) (IDDF2021-ABS-0070 Figure 3. ROC curves for HAP score and ALBI grade), indicating a significant performance of HAP score compared with ALBI grade in prognosis of HCC treated with TACE.

Conclusions HAP is a useful score to assist for the management decisions of patients with HCC requiring TACE due to its value in predicting mortality and survival.

\section{IDDF2021-ABS-0071 TUMOR BURDEN SCORE AS A NEW PROGNOSTIC MARKER FOR PATIENTS WITH HEPATOCELLULAR CARCINOMA UNDERGOING TRANSARTERIAL CHEMOEMBOLIZATION}

${ }^{1}$ Shu-Yein Ho*, ${ }^{2}$ Chih-Chieh Ko, ${ }^{2}$ Chien-Wei Su, ${ }^{3}$ Teh-la Huo. ${ }^{1}$ Division of Gastroenterology and Hepatology, Min-Sheng General Hospital, Taoyuan, Taiwan; ${ }^{2}$ Department of Medicine, Taipei Veterans General Hospital, Taipei, Taiwan; ${ }^{3}$ Department of Medical Research, Taipei Veterans General Hospital, Taipei., Taiwan

\subsection{6/gutjnl-2021-IDDF.78}

Background Tumor size and numbers are major determinants of tumor burden in hepatocellular carcinoma (HCC). Patients with HCC undergoing transarterial chemoembolization (TACE) have variables outcomes due to heterogeneity of tumor burden. Recently, tumor burden score (TBS) was proposed to evaluate the extent of tumor involvement. However, the prognostic accuracy of TBS has not been evaluated in HCC. This study aimed to assess its prognostic role in HCC patients undergoing TACE.

Methods A total of 935 treatment-naive HCC patients receiving TACE were retrospectively analyzed. Multivariate Cox proportional hazard model was used to determine independent prognostic predictors.TBS was defined as the distance from the origin of the Cartesian plane and the comparison of two variables: maximum tumor size (X-axis) and number of tumors (Y-axis) so that $\mathrm{TBS}^{2}=(\text { maximum tumor diameter })^{2}+$ (number $^{2}$ of tumors $)^{2}$. Patients have divided accordingly into three groups:high TBS (over 13.74), medium TBS (3.36-13.74), and low TBS (less than 3.36).

Results TBS tended to increase with the increasing size and number of tumors in this study (IDDF2021-ABS-0071 Figure 1. The association of TBS with tumor diameter and numbers). The Cox model showed that serum creatinine $\geq 1.2 \mathrm{mg} /$ dl (hazard ratio(HR):1.296,95\% confidence interval $(\mathrm{CI}): 1.077-1.559, \mathrm{p}=0.006), \quad$ serum $\quad \alpha$-fetoprotein $\geq 400 \mathrm{ng} / \mathrm{dl}$ (HR:2.245,95\%CI:1.905-2.465, p<0.001), vascular invasion (HR:1.870, 95\% CI: 1.520-2.301,p<0.001), medium TBS (HR:1.489,95\%CI:1.206-1.839, p<0.001), high TBS (HR:2.563,95\%CI:1.823-3.602,p<0.001), albumin-bilirubin (ALBI) grade 2-3 (HR:1.521,95\% CI:1.291-1.792,p<0.001),

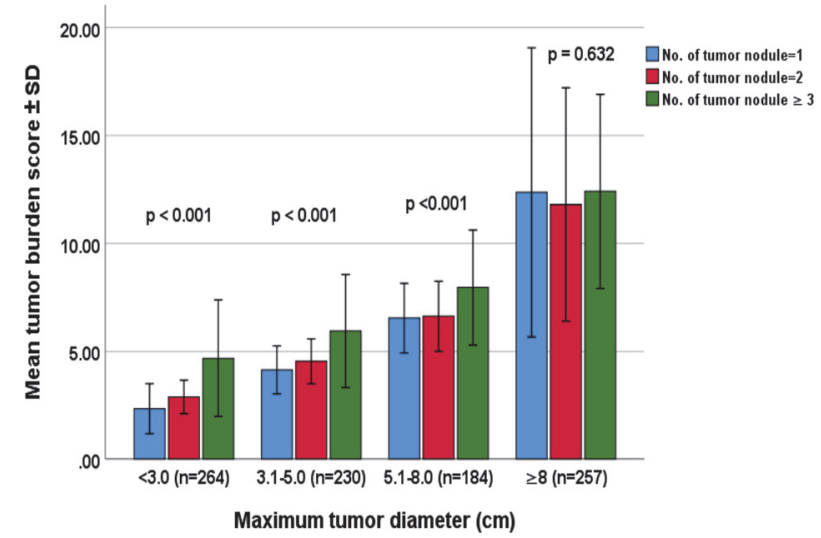

Abstract IDDF2021-ABS-0071 Figure 1 